\title{
Does cerebrospinal fluid analysis have a meaningful role in the diagnosis of CIDP?
}

Zachary N. London MD, University of Michigan Department of Neurology

Dustin G. Nowacek MD, University of Michigan Department of Neurology

Corresponding Author:

Zachary N. London MD

1324 Taubman Center

1500 E. Medical Center Dr.

Ann Arbor, MI 48109

Word count: 1415

Acknowledgements: None

Running Title: CSF's role in CIDP

Ethical Publication Statement. We confirm that we have read the Journal's position on issues involved in ethical publication and affirm that this report is consistent with those guidelines

Disclosures: None

Key words: Chronic inflammatory demyelinating polyradiculoneuropathy (CIDP), Cerebrospinal fluid (CSF), Intravenous immunoglobulin (IVIg), Neuropathy, Lumbar puncture, Normative Data

This is the author manuscript accepted for publication and has undergone full peer review but has not been through the copyediting, typesetting, pagination and proofreading process, which may lead to differences between this version and the Version of Record. Please cite this article as doi: $10.1002 /$ mus.26513

This article is protected by copyright. All rights reserved. 
One of the more satisfying professional experiences is helping a neurologically disabled patient regain strength and function. Chronic inflammatory demyelinating polyradiculoneuropathy (CIDP) is a neuropathy syndrome for which there are effective disease-modifying treatments. It is not surprising, then, that physicians can be tenacious in the pursuit of diagnostic testing for CIDP, and quick to prescribe intravenous immunoglobulin (IVIg) and other therapies when it is suspected.

In the absence of a specific biomarker, the diagnosis of CIDP relies on a combination of clinical features and ancillary tests, none of which is adequate in isolation. At least 17 sets of diagnostic criteria have been published, giving physicians substantial leeway when it comes to making the diagnosis. ${ }^{1}$ In an effort to improve consistency, more experts have been advocating for physicians to utilize the revised European Federation of Neurological Societies/Peripheral Nerve Society (EFNS/PNS) diagnostic criteria for CIDP. ${ }^{2}$ These commonly-accepted consensus-derived criteria encompass both typical and atypical CIDP phenotypes, and rely on clinical, electrophysiologic, laboratory, imaging, and pathologic findings to classify definite, probable, and possible CIDP. Applying the criteria for possible CIDP, which have a sensitivity of $91.1 \%$ and a specificity of $65.8 \%$, casts the widest net for patients who may warrant a therapeutic trial of IVIg or another immunomodulatory agent. ${ }^{3}$

CIDP is overdiagnosed. $47 \%$ of patients referred to a tertiary care neuromuscular practice with the diagnosis of CIDP did not meet EFNS/PNS criteria for even possible CIDP. ${ }^{4}$ In another study, only $32.2 \%$ of 248 patients receiving IVIg for an immune-mediated neuropathy, including but not limited to CIDP, 
were thought to be appropriate for IVIg therapy. ${ }^{5}$ Even this may be an overestimation, because the study did not include insurer-denied cases. In a study of patients treated by community neurologists for CIDP, only $11 \%$ of those receiving IVIg had documentation sufficient enough to meet EFNS/PNS criteria for at least possible CIDP. ${ }^{6}$

Our propensity to overdiagnose CIDP is not without risk. Physicians may anchor on the wrong diagnosis rather than continuing to explore other diagnoses that would be managed differently. This can lead to the inappropriate use of expensive and potentially toxic drugs. In one study, patients misdiagnosed with CIDP were treated with IVIg for an average of 1.5 years. ${ }^{4}$ Patients also may anchor on the wrong diagnosis. When a misdiagnosed patient has subjective benefit from therapy, efforts to discontinue the inappropriate treatment may strain the physician-patient relationship or contribute to psychological distress in the patient.

There are clear patterns to the ways in which physicians overdiagnose CIDP. One of the most common is over-reliance on patient-reported perceptions of treatment benefit. The placebo effect of IVIg can be so high that patients with CIDP and those misdiagnosed with CIDP report similar benefits from treatment. ${ }^{4}$ When objective measures of strength are used, patients correctly diagnosed with CIDP are much more likely to improve than those incorrectly diagnosed.

This article is protected by copyright. All rights reserved. 
Another common factor that leads to the misdiagnosis of CIDP is over-interpretation of demyelinating features on nerve conduction studies. This includes misclassification of mild conduction velocity slowing in motor nerves with a low compound muscle action potential amplitude, or in patients with a condition known to cause mild conduction slowing, such as diabetes mellitus. Other common pitfalls include misidentifying slowing across a common entrapment site as a sign of a generalized or multifocal demyelinating disorder, or overcalling conduction block or slowing that is more likely due to technical factors. ${ }^{7}$

Putting too much stock in mildly to moderately elevated cerebrospinal fluid (CSF) protein levels can also lead to the misdiagnosis of CIDP. The EFNS/PNS criteria for CIDP list "elevated protein" in the CSF as a supportive criterion, but do not define that further. While most clinical laboratories use an upper reference limit (URL) of $45 \mathrm{mg} / \mathrm{dL}$ to define elevated protein, there is emerging evidence to suggest that higher, age-matched URL should be implemented $(50 \mathrm{mg} / \mathrm{dL}$ for patients $<50$ and $60 \mathrm{mg} / \mathrm{dL}$ for patients over age 50$).^{8}$

In this issue of Muscle \& Nerve, Breiner et al applied different sets of criteria with higher protein URLs to patient cohorts that were either correctly or incorrectly diagnosed with CIDP. ${ }^{9}$ CSF protein elevation was found in both CIDP and misdiagnosed CIDP groups, but the misdiagnosed group tended to have more mild elevations (median $53 \mathrm{mg} / \mathrm{dL}$ ) compared to the correctly diagnosed group (mean $105 \mathrm{mg} / \mathrm{dL}$ ).

This article is protected by copyright. All rights reserved. 
Applying the higher URL led to a decline in false-positive results without changing the overall detection rate of CIDP using EFNS/PNS criteria.

To understand why the CIDP detection rate did not change with the higher URLs, it is important to appreciate the limited role that elevated CSF protein has in the EFNS/PNS criteria for CIDP. All of the correctly-diagnosed CIDP patients in this study met clinical and electrodiagnostic criteria, which are mandatory for all three diagnostic categories - definite, probable, and possible CIDP. Elevated CSF protein is considered a supportive criterion for CIDP. Other supportive criteria include an objective response to therapy, or specific abnormalities on MRI, somatosensory evoked potentials, or nerve biopsy. Supportive criteria, when present, may differentiate among the three CIDP categories, but are not used to distinguish possible CIDP from not CIDP. Thus, if one is willing to offer immunomodulatory therapy to any patient with possible CIDP or better, CSF analysis would have no role in treatment decisions.

If clinical decision making depended on categorizing CIDP, rather than just identifying the presence or absence of CIDP, CSF analysis would have a theoretical value. In the EFNS/PNS criteria, the presence of elevated CSF protein can move a patient from possible to probable or probable to definite CIDP. In the study by Breiner et al, 43 patients who were correctly diagnosed with CIDP underwent CSF analysis. Within this cohort, an elevated CSF protein level was the sole supportive criterion in only 1 case. Consequently, CSF analysis did not even have the potential to influence the categorization of CIDP in 
$97 \%$ of cases. This suggests that the role of CSF analysis in CIDP may be low, even if a physician considers CIDP categorization as a factor in medical decision making.

Further study is needed to know if the rigid application of EFNS/PNS criteria correlate with disease activity, treatment response and outcome. The international CIDP outcome study (ICOS) is following treatment outcomes in a cohort of patients that meet standard EFNS/PNS criteria, and another cohort of patients that meet clinical and supportive criteria without electrodiagnostic criteria. ${ }^{10}$ If the study finds evidence of treatment effect in the latter group, the value of CSF analysis in the initial diagnosis of CIDP may be augmented.

No matter the results of the ICOS study, the assertion that lumbar puncture has limited value in the work-up of CIDP needs to be balanced with several caveats. The EFNS/PNS clinical criteria for CIDP rely on the physician's evaluations of strength, deep tendon reflexes, and time course of symptoms. These are subject to human interpretation, and borderline findings may lead to diagnostic uncertainty. In these cases, when rigid application of the EFNS/PNS criteria may not feasible, elevated CSF protein levels may sway the physician towards a CIDP diagnosis. Other supportive criteria may also have value in these cases, but CSF analysis may be more widely available than spine MRI, evoked potential studies, and nerve biopsy. Finally, CSF analysis may include measurements of white blood cells and other constituents that aid in screening for infectious or neoplastic processes that can mimic CIDP or other neuropathy phenotypes. 
This study informs the discussion about the misdiagnosis of CIDP. CSF analysis is probably overutilized in the routine evaluation of CIDP and may contribute more to the misdiagnosis than the correct diagnosis of CIDP. In the absence of supportive clinical and electrodiagnostic data, the specificity of elevated CSF protein is low, and the primary value of CSF analysis is to rule out alternative diagnoses. When patients meet minimal mandatory criteria for CIDP, elevated CSF protein is a supportive criterion that may help further categorize the diagnosis. In this context, standardized, age-matched evidence-based reference values for CSF protein should be adopted by clinicians and clinical laboratories.

There are other ways to reduce the misdiagnosis of CIDP. Foremost, we need to improve utilization of and adherence to CIDP diagnostic guidelines, with particular attention to correct interpretation of electrodiagnostic data. Neuromuscular physicians should become proficient in applying diagnostic criteria, and offer expert consultation for equivocal cases. When patients are treated for CIDP, there need to be serial clinical evaluations to look for objective indicators of treatment efficacy. This will allow us to limit the overutilization of expensive, scarce, and potentially harmful resources.

1. Peltier AC, Donofrio PD. Chronic inflammatory demyelinating polyradiculoneuropathy: from bench to bedside. Seminars in neurology. 2012;32(3):187-195.

2. Van den Bergh PY, Hadden RD, Bouche P, et al. European Federation of Neurological Societies/Peripheral Nerve Society guideline on management of chronic inflammatory demyelinating polyradiculoneuropathy: report of a joint task force of the European Federation of Neurological Societies and the Peripheral Nerve Society - first revision. Eur J Neurol. 2010;17(3):356-363.

This article is protected by copyright. All rights reserved. 
3. Breiner A, Brannagan TH, 3rd. Comparison of sensitivity and specificity among 15 criteria for chronic inflammatory demyelinating polyneuropathy. Muscle \& nerve. 2014;50(1):40-46.

4. Allen JA, Lewis RA. CIDP diagnostic pitfalls and perception of treatment benefit. Neurology. 2015;85(6):498-504.

5. Levine TD, Katz JS, Barohn R, et al. Review process for IVIg treatment: Lessons learned from INSIGHTS neuropathy study. Neurol Clin Pract. 2018;8(5):429-436.

6. Allen JA, Gorson KC, Gelinas D. Challenges in the diagnosis of chronic inflammatory demyelinating polyneuropathy. Brain and behavior. 2018;8(3):e00932.

7. Cornblath DR, Gorson KC, Hughes RA, Merkies IS. Observations on chronic inflammatory demyelinating polyneuropathy: A plea for a rigorous approach to diagnosis and treatment. Journal of the neurological sciences. 2013;330(1-2):2-3.

8. Breiner A, Moher D, Brooks J, et al. Adult CSF total protein upper reference limits should be agepartitioned and significantly higher than $0.45 \mathrm{~g} / \mathrm{L}$ : a systematic review. Journal of neurology. 2019;266(3):616-624.

9. Breiner A, Bourque PR, Allen JA. Updated Cerebrospinal Fluid Total Protein Reference Values Improve CIDP Diagnosis. Muscle \& nerve. 2019.

10. Bunschoten C, Eftimov F, van der Pol WL, Jacobs BC, Consortium I. International chronic inflammatory demyelinating polyneuropathy outcome study (ICOS): Protocol of a prospective observational cohort study on clinical and biological predictors of disease course and outcome. Journal of the peripheral nervous system : JPNS. 2019;24(1):34-38. 